\title{
BMJ Open Effectiveness of topical and ablative therapies in treatment of anogenital warts: a systematic review and network meta-analysis
}

\author{
Samantha Barton, ${ }^{1}$ Victoria Wakefield, ${ }^{1}$ Colm O'Mahony, ${ }^{2,3}$ Steven Edwards ${ }^{1}$
}

To cite: Barton S, Wakefield V, O'Mahony C, et al. Effectiveness of topical and ablative therapies in treatment of anogenital warts: a systematic review and network meta-analysis. BMJ Open 2019;9:e027765. doi:10.1136/ bmjopen-2018-027765

- Prepublication history and additional material for this paper are available online. To view please visit the journal (http:// dx.doi.org/10.1136/bmjopen2018-027765).

Received 13 November 2018 Revised 10 September 2019 Accepted 12 September 2019

Check for updates

(C) Author(s) (or their employer(s)) 2019. Re-use permitted under CC BY-NC. No commercial re-use. See rights and permissions. Published by BMJ.

${ }^{1}$ BMJ-Technology Assessment Group, London, UK

${ }^{2}$ Nuffield Health, Chester, UK

${ }^{3}$ Liverpool Medical Institution, Liverpool, UK

Correspondence to

Dr Samantha Barton;

samantha.barton@bmj.com

\section{ABSTRACT}

Objective To generate estimates of comparative clinical effectiveness for interventions used in the treatment of anogenital warts (AGWs) through the systematic review, appraisal and synthesis of data from randomised controlled trials (RCTs).

Design Systematic review and network meta-analysis of RCTs. Search strategies were developed for MEDLINE, Embase, the Cochrane Library and the Web of Science. For electronic databases, searches were run from inception to March 2018. The systematic review was carried out following the general principles recommended in the Preferred Reporting Items for Systematic Reviews and Meta-Analyses statement.

Participants People aged $\geq 16$ years with clinically diagnosed AGWs (irrespective of biopsy confirmation). Interventions Topical and ablative treatments recommended by the British Association for Sexual Health and HIV for the treatment of AGWs, either as monotherapy or in combination versus each other.

Outcome measures Complete clearance of AGWs at the end of treatment and at other scheduled visits, and rate of recurrence.

Results Thirty-seven RCTs met inclusion criteria. Twenty studies were assessed as being at unclear risk of bias, with the remaining studies categorised as high risk of bias. Network meta-analysis indicates that, of the treatment options compared, carbon dioxide laser therapy is the most effective treatment for achieving complete clearance of AGWs at the end of treatment. Of patient-applied topical treatments, podophyllotoxin $0.5 \%$ solution was found to be the most effective at achieving complete clearance, and was associated with a statistically significant difference compared with imiquimod 5\% cream and polyphenon E 10\% ointment $(p<0.05)$. Few data were available on recurrence of AGWs after complete clearance. Of the interventions evaluated, surgical excision was the most effective at minimising risk of recurrence.

Conclusion of the studies assessed, as a collective, the quality of the evidence is low. Few studies are available that evaluate treatment options versus each other. Trial registration number CRD42013005457

\section{INTRODUCTION}

In 2013, global annual incidence of anogenital warts (AGWs) was estimated to range
Strengths and limitations of this study

This review addresses uncertainty in a disease area in which further research is unlikely.

- Systematic approach taken to assess the literature.

- The strength of the conclusions that can be drawn is weakened by uncertainty around the quality of the evidence base evaluating treatment options for anogenital warts.

between 160 and 289 per 100000 , with incidence peaking in men aged 25 to 29 years and women aged $<24$ years. ${ }^{1}$ Arising from infection with human papillomavirus (HPV), predominantly types 6 and 11, AGWs are the most commonly diagnosed viral sexually transmitted infection. ${ }^{1}$ AGWs can potentially clear without treatment, most likely in people who are immunocompetent, and some people may prefer to wait a period of time before starting treatment.

Other subtypes of HPV are associated with an increase in risk of developing anogenital cancers. Subtypes 16 and 18 are linked with increased risk of cervical cancer in women and account for an estimated $70 \%$ of all cervical cancers. ${ }^{2}{ }^{3}$ In 2008, the UK Department of Health announced the introduction of an HPV immunisation programme against HPVs for girls aged 12 to 13 years starting in September of that year. ${ }^{4}$ Two vaccines against infection with HPVs are available in the UK, a bivalent vaccine (Cervarix; GlaxoSmithKline) protecting against subtypes 16 and 18 , and a quadrivalent vaccine (Gardasil; Sanofi Pasteur) that additionally protects against subtypes 6 and 11. Economical evaluation of the two vaccines led to the bivalent vaccine being chosen for the first 3 years of the immunisation programme. ${ }^{4}$ However, the UK Department of Health approved the use of the quadrivalent vaccine in 2012, and, in 2018, extended HPV vaccination to include 
boys aged 12 to 13 years, starting in $2019 .{ }^{5}$ Given approval for use of the quadrivalent vaccine, it is expected that there will soon be a reduction in the number of cases of AGWs presenting at sexual health clinics equivalent to that observed in other countries. In Australia, which was one of the first countries to introduce a national vaccination programme with the quadrivalent vaccine, assessment of six sexual health clinics identified a considerable decline in the proportion of women aged under 21 years presenting with AGWs at the clinics, dropping from $11.5 \%$ in 2007 to $0.85 \%$ in $2011(\mathrm{p}<0.001){ }^{6}$

Although a national HPV vaccination programme in the UK will reduce the number of people presenting with AGWs, not all women will receive the vaccination and, at this time, boys are not vaccinated. The need for effective treatments against AGWs remains. Treatments for AGWs available in the UK encompass clinician-applied destructive techniques and patient-applied topical treatments. ${ }^{7}$ Topical treatments that can be applied by the person with AGWs at home are imiquimod 5\% cream and podophyllotoxin (available as a $0.5 \%$ solution and a $0.15 \%$ cream). Other topical treatment options applied by a clinician are trichloroacetic acid (TCAA) and podophyllin, although the use of podophyllin is no longer recommended by British Association for Sexual Health and HIV (BASHH). Patient-applied topical treatments are increasingly prescribed, particularly for mild, early lesions, because of the convenience of use and the increased privacy for the patient. ${ }^{7}$

Ablative methods that require administration by a clinician, such as electrosurgery (cautery, hyfrecation), cryotherapy and laser therapy act to debulk the visible lesions. Alternatively, a clinician may decide to use a combination of a topical treatment and an ablative therapy. ${ }^{7}$ People who are immunocompromised typically have a poorer response to the treatments available. The topical antiviral cream cidofovir (Vistide; Gilead), which is primarily used to treat cytomegalovirus retinitis, has been investigated as a potential treatment option for those with AGWs who are immunocompromised. ${ }^{8-10}$

Treatment choice is typically decided after discussion between the treating clinician and the person with AGWs. A systematic review carried out in 2014 evaluating the clinical and cost-effectiveness of clinician and patientapplied treatments implemented network meta-analyses (NMAs) and identified that ablative techniques were typically more effective than topically applied interventions at completely clearing AGWs. ${ }^{11}$ In 2015, BASHH updated their guidance on management of AGWs to include Catephen (polyphenon E) 10\% ointment. ${ }^{7}$ A systematic review focusing on topical treatments found that imiquimod $3.75 \%$ and $5 \%$ cream, podophyllotoxin $0.5 \%$ solution and gel and polyphenon E $10 \%$ and $15 \%$ ointment were more effective than placebo, but, because of the low quality of the identified evidence definitive conclusions were not possible. ${ }^{12}$ The review did not carry out a NMA. The research presented here is an update of the systematic review comparing ablative and topical treatments, ${ }^{11}$ and has been expanded to include polyphenon E $10 \%$ ointment. Topical interventions assessed are limited to those recommended by BASHH.

\section{METHODS}

Electronic databases were searched (Ovid MEDLINE In-Process \& Other Non-Indexed Citations, Ovid Embase and Cochrane Library) from inception to March 2018. Web of Science was searched from 2000 to March 2018. Search strategies were designed to include medical subject headings (MeSH) and text terms for AGWs, including 'condyloma acuminata' (the medical term for AGWs), and were tailored to each database. MeSH or text terms for interventions of interest were not included in the original search strategies. For the review reported here, the original searches were updated and new strategies devised to retrieve potentially relevant records of studies evaluating polyphenon E 10\% ointment. Search filters designed to retrieve records based on study design were identified through the InterTASC Information Specialists' Sub-Group search filter resource. ${ }^{13}$ Full details of the search strategy used to retrieve records from Ovid Embase is provided in online supplementary information 1. Bibliographies of previous overviews, guidelines and retrieved articles were manually reviewed for additional studies. Two researchers (SB and one of CK, NM or VW) independently screened the titles and abstracts returned by the searches according to the prespecified eligibility criteria. In cases in which consensus could not be achieved, the full texts of potentially relevant studies were ordered. Two reviewers (SB and one of VW and CK) independently assessed full publications for inclusion. Discrepancies were resolved by discussion, with involvement of a third reviewer (SE) if consensus could not be reached. Methods for the review are reported in more detail in the published protocol (CRD42013005457) and were based on the principles published by the National Health Service Centre for Reviews and Dissemination. ${ }^{14}$

\section{Patient and public involvement}

Patients were not directly involved in the development of the review update.

\section{Inclusion criteria}

Randomised controlled trials (RCTs) evaluating treatment of patients aged $\geq 16$ years with clinically diagnosed AGWs (irrespective of biopsy confirmation) were included. The interventions of interest were topical treatments and ablative techniques, either alone or in combination. Topical treatments assessed were: podophyllotoxin $0.5 \%$ and $0.3 \%$ (both solution and cream), imiquimod 5\% cream, TCAA, polyphenon E 10\% and $15 \%$ (ointment) and cidofovir. Physical ablation methods evaluated were: cryotherapy (liquid nitrogen spray or cryoprobe), surgical excision (under local anaesthetic), electrotherapy (electrocautery, hyfrecator surgery) and laser therapy. The primary outcomes of interest to the 
review were AGW clearance, both at completion of treatment (eg, up to 16 weeks for imiquimod) and at later time points after completion of treatment (eg, 3 months, 6 months) and recurrence rate. Other outcomes assessed were: time to complete clearance, volume of wart clearance (e.g. $>50 \%$ clearance of original AGWs or $>75 \%$ clearance of original AGWs), relief of symptoms during treatment, appearance of new warts during treatment, quality-of-life (QoL) as reported using a validated QoL rating scale (eg, EQ-5D, SF-36), adverse events and malignancy. RCTs were included if the interventions of interest were evaluated in a population with AGWs and compared with each other (alone or in combination), placebo or no treatment. Studies were excluded if none of the outcomes of interest was reported.

\section{Data abstraction and quality assessment}

After an initial pilot data extraction phase of 10 studies by two reviewers (SB and VW), during which the suitability of the standardised data extraction form was evaluated, one reviewer (various) extracted data from the remaining studies into a modified data extraction form, with validation of the data by a second reviewer $(\mathrm{SB})$ : the data extraction form is available in online supplementary information 2. Information extracted included details on study design and methodology, the baseline characteristics of the population and data on outcomes of interest. Discrepancies were resolved by discussion, with involvement of a third reviewer (SE) when necessary. In cases where limited information was reported, authors were contacted with a request for additional detail. Two reviewers independently assessed the quality of the clinical effectiveness studies (SB and one of various reviewers). Discrepancies were resolved by discussion, with involvement of a third reviewer when necessary. Study quality was recorded using the Cochrane risk of bias tool. ${ }^{15}{ }^{16}$ Outcome-specific risk of bias was determined for the outcomes for which data were extracted. ${ }^{15}$ The three bias assessment categories used were low, unclear and high. A study was assessed as being at low risk of bias when all domains were associated with low risk of bias. Assessment of at least one domain as high risk of bias led to overall rating as high risk of bias. In cases where no domain was assessed as high risk, but one domain was unclear, the overall risk of bias for the study was determined to be unclear.

\section{Meta-analysis}

The comparative clinical effectiveness of interventions was investigated using NMAs. The methods used for NMA followed the guidance described in the National Institute for Health and Care Excellence Decision Support Unit's Technical Support Documents for Evidence Synthesis. ${ }^{17} 18$ NMAs were conducted using a Bayesian Markov chain Monte Carlo simulation in WinBUGS (V.1.4; MRC Biostatistics Unit, Cambridge, UK). To ensure convergence on the posterior distribution, analyses were based on 60000 iterations after a 'burn in' of 60000 iterations. Uniformed priors were implemented and no covariates were accounted for in the NMA. Fixed and random effects models were used for the primary and sensitivity analyses with the best fitting model chosen based on the deviance information criterion. To facilitate inclusion of interventions of interest, the network was extended to include podophyllin $20 \%$ to $25 \%$. Data from multi-arm studies were adjusted to account for correlations in relative treatment effects. ${ }^{19}$ Effect estimate for dichotomous outcomes was OR, with accompanying 95\% credible interval (95\% CrI) using people as the unit of analysis. The probability of a treatment being the most effective at achieving complete clearance at the end of treatment was assessed using surface under the cumulative ranking (SUCRA).$^{20}$ A $95 \%$ CrI can be interpreted as a $95 \%$ probability that the parameter falls within this range. If a $95 \%$ CrI does not include one this can, therefore, be interpreted as a statistically significant result (at the $5 \%$ level of significance). Analyses for clinical outcomes were based on studies of low, or unclear risk of bias. Sensitivity analyses included studies deemed to be at high risk of bias. Ablative techniques, such as cryotherapy and surgical excision, are widely recognised as being the most effective techniques to achieve complete clearance of AGWs and, in RCTs, are often applied until complete clearance is achieved. To evaluate comparative clinical effectiveness of treatments administered over a set time frame, studies evaluating ablative techniques applied until complete clearance were not included in the NMA for analysis of complete clearance at the end of treatment. Studies evaluating imiquimod $5 \%$ cream were included if treatment was applied three times per week for a prespecified duration. Imiquimod $5 \%$ cream can be applied for up to a maximum of 16 weeks per episode of AGWs. RCTs of any prespecified duration of treatment were included in the NMA.

\section{RESULTS}

Appraisal of the searches run in September 2014 for an earlier systematic review of clinical and costeffectiveness ${ }^{11}$ resulted in the identification of 70 relevant articles describing 60 RCTs (figure 1). Re-assessment of the full text publications in line with the revised protocol led to the exclusion of 27 RCTs described in 37 articles. An update search identified five new RCTs (figure 1), of which four are included in the review, to give a total of 37 RCTs: characteristics of all included studies are available as online supplementary information 3, complete data extraction forms for the four studies added at update are provided in online supplementary information 4 and a list of studies excluded during review of full text is available in online supplementary information 5. A translation could not be obtained for one RCT published in Farsi that treated 30 women with cryotherapy and 30 women with podophyllin $25 \% .{ }^{21}$ From the study abstract, it is unclear whether the study was truly randomised and whether the results are consistent with other studies evaluating cryotherapy versus podophyllin $25 \%$. 
Original searches Sept 2014
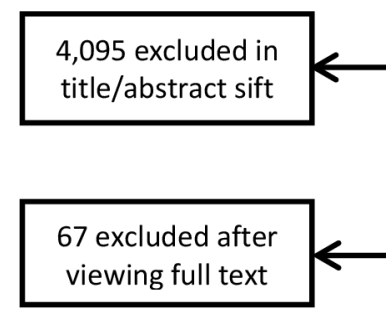

RCT search $=6,085$ 1,853 duplicates

4,232 screened

137 full texts assessed

70 included articles (describing 60 RCTs)

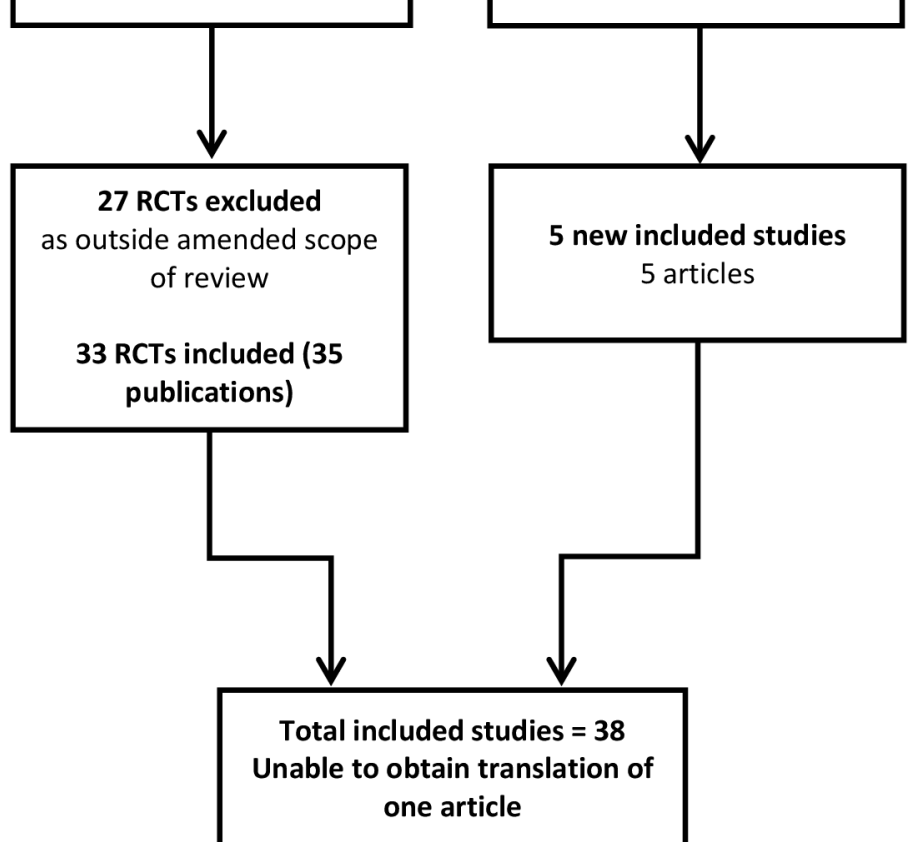

Update searches Mar 2018

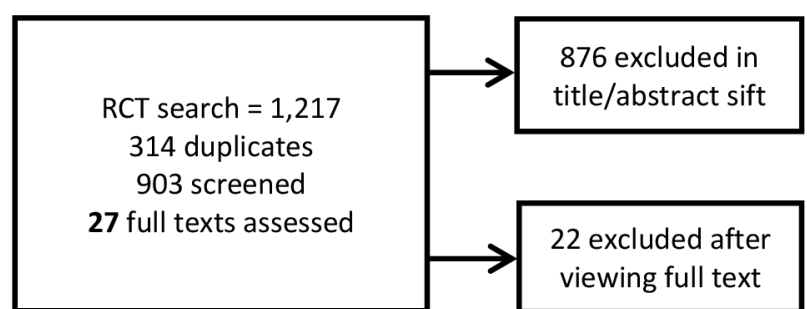

Figure 1 Preferred Reporting Items for Systematic Reviews and Meta-Analyses diagram for original and update search. RCT, randomised controlled trial.

All studies enrolled people with AGWs, with most studies focusing on treatment of external AGWs: of the studies included in the NMA, three RCTs enrolled people with internal and external AGWs. ${ }^{922}{ }^{23}$ Considering HIV status, only two studies included in the NMA enrolled those who were HIV-positive, ${ }^{94}$ with the remaining studies including people who were immunocompetent or not specifying whether HIV-status was assessed during recruitment. There was considerable variation across studies in eligibility criteria in terms of size and volume of AGWs. Some studies required only clinical diagnosis of AGWs for eligibility, whereas others specified a minimum and maximum number of AGWs, or a minimum AGW total surface area for inclusion (online supplementary information 2). Study size ranged from 12 people in the smallest study ${ }^{8}$ to 503 in the largest identified study. ${ }^{25}$

In studies assessing ablative techniques, such as cryotherapy or electrocautery, one study evaluating electrotherapy and placebo in which active intervention was given until complete clearance of AGWs was excluded from the NMA assessing complete clearance at the end of treatment. ${ }^{22}$ Across studies evaluating topical interventions, treatment application schedule and duration of treatment were specified for patient-applied topical treatments, including imiquimod 5\% cream and podophyllotoxin $0.15 \%$ cream. Studies evaluating topical treatments at the licensed dosing schedule were included in the review of clinical effectiveness.

Treatments compared directly in each of the identified studies, together with information on whether the study has been included in a NMA are presented in table 1 .

Of the 37 identified RCTs, no study was deemed to be at an overall low risk of bias (online supplementary information 2, table 1). Twenty studies were considered to have unclear risk of bias, with limited reporting in the full publication on the methods implemented for random sequence generation, and concealment of allocation, together with lack of clarity on level of masking, being the predominant reason for categorisation of unclear risk of bias. Reported lack of masking of key trial personnel was the most common reason for assessment of high-risk of bias for the remaining 17 studies. Primary outcomes of the review are complete clearance of AGWs and recurrence, both of which are subjective in nature and open to assessment bias if the investigator is aware of treatment allocation. A key secondary outcome assessed is complete clearance at time points after the end of treatment, which might be of particular interest to those experiencing AGWs. Although it may not be feasible to mask key trial personnel to treatment allocation in studies comparing ablative versus topical treatments, masking 
Table 1 Direct comparisons evaluated in identified RCTs

\begin{tabular}{|c|c|c|}
\hline $\begin{array}{l}\text { Direct comparisons relevant to scope included in any NMA (primary } \\
\text { or sensitivity for any outcome) }\end{array}$ & $\begin{array}{l}\text { Identitied studies e } \\
\text { Included in a NMA }\end{array}$ & Omitted from NMA \\
\hline \multicolumn{3}{|l|}{ vs placebo } \\
\hline Imiquimod 5\% cream & $\begin{array}{l}\text { Arican }^{35} \\
\text { Beutner }^{36} \\
\text { Edwards }^{37} \\
\text { Gilson }^{24} \\
\text { Tyring }^{38}\end{array}$ & - \\
\hline Polyphenon E 10\% ointment & $\begin{array}{l}\text { Stockfleth } \\
\text { Tatti }^{25}\end{array}$ & - \\
\hline Podophyllotoxin $0.5 \%$ solution & $\begin{array}{l}\text { Beutner }^{40} \\
\text { Kirby }^{41} \\
\text { Von Krogh }^{42}\end{array}$ & Greenberg $^{43}$ \\
\hline Podophyllotoxin $0.5 \%$ solution vs podophyllotoxin $0.15 \%$ cream & $\begin{array}{l}\text { Lacey }^{23} \\
\text { Strand }^{44}\end{array}$ & Claesson $^{45}$ \\
\hline Podophyllotoxin $0.5 \%$ solution vs imiquimod $5 \%$ cream & Komericki $^{46}$ & - \\
\hline
\end{tabular}

Topical treatment vs ablative treatment

Imiquimod 5\% cream vs cryotherapy

$\begin{array}{ll}\text { Akhavan }^{29} & \text { Tuncel }^{47}\end{array}$

Stefanaki $^{28}$

TCAA 80\%-90\% versus cryotherapy

Abdullah $^{48}$

Ablative treatments vs each other

Cryotherapy vs electrotherapy

Stone ${ }^{49}$

Cryotherapy vs $\mathrm{CO}_{2}$ laser therapy

Azizjalali ${ }^{50}$

Simmons $^{27}$

Argon plasma coagulation vs electrotherapy

$\begin{array}{ll}- & \text { Braga }^{51} \\ - & \text { Ferenczy }^{52}\end{array}$

$\mathrm{CO}_{2}$ laser therapy vs electrotherapy

Orlando $^{9}$

Stone ${ }^{49}$

Cidofovir vs electrotherapy

Gilson $^{26}$

Combination treatment vs monotherapy

Cryotherapy plus podophyllotoxin $0.15 \%$ cream vs cryotherapy alone

Argon plasma coagulation plus imiquimod $5 \%$ vs argon plasma

- $\quad$ Viazis $^{53}$
coagulation alone

Additional comparisons included to strengthen NMA

Podophyllotoxin $0.5 \%$ solution vs podophyllin $20 \%-25 \%$

Identified studies evaluating comparison

Edwards ${ }^{54}$

$\mathrm{Kar}^{31}$

Lacey $^{23}$

Lassus $^{32}$

Mazurkiewicz ${ }^{55}$

Podophyllotoxin $0.15 \%$ cream vs podophyllin 20\%-25\%

Lacey $^{23}$

Imiquimod $5 \%$ cream vs podophyllin $20 \%-25 \%$

Akhavan $^{29}$

Padhiar $^{33}$

Cryotherapy vs podophyllin 20\%-25\%

Akhavan $^{29}$

Sherrard $^{56}$

Stone ${ }^{49}$

Surgical excision vs podophyllin 20\%-25\%

Jensen $^{30}$

Electrotherapy vs podophyllin 20\%-25\%

Stone ${ }^{49}$

$\mathrm{CO}_{2}$, carbon dioxide; NMA, network meta-analysis; RCT, randomised controlled trial; TCAA, trichloroacetic acid. 
a) Primary analysis of complete clearance at end of treatment

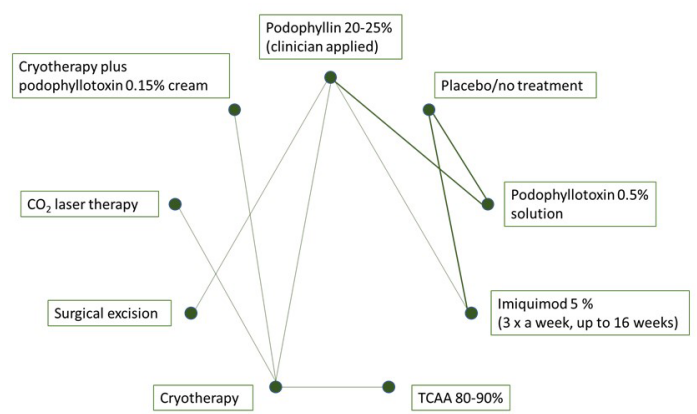

b) Sensitivity analysis of complete clearance at end of treatment

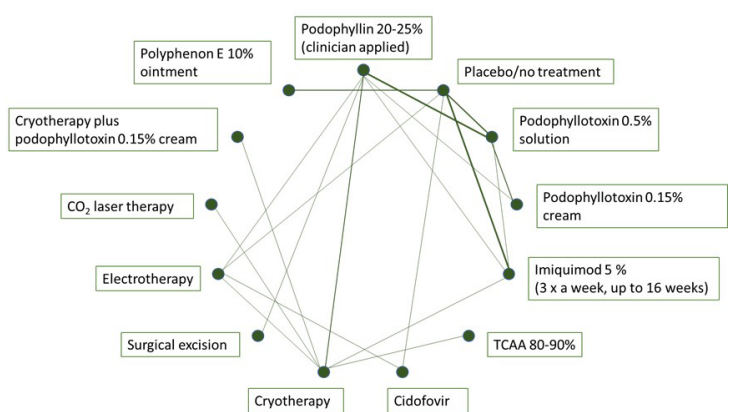

Figure 2 Networks for primary and sensitivity analyses of complete clearance of anogenitalwarts at end of treatment. $\mathrm{CO}_{2}$, carbon dioxide; TCAA, trichloroacetic acid.

the clinician(s) assessing outcomes would minimise the risk of bias for this domain.

\section{Complete clearance}

Fifteen studies formed the primary network on complete clearance of AGWs at the end of treatment and generated effect estimates for nine interventions and 36 comparisons (figure 2). By contrast, 27 studies were included in the sensitivity analysis, generating information on 13 interventions and 78 comparisons (figure 2). Analysis of model fit identified the random effects model to be the best fitting in the primary and the sensitivity analyses. Reporting of results focuses on effect estimates versus podophyllotoxin $0.5 \%$ solution for treatments recommended in the BASHH guidelines for management of
AGWs (table 2). NMA indicated podophyllotoxin $0.5 \%$ solution to be statistically significantly more effective than imiquimod 5\% cream (primary analysis: OR $<1$ favours podophyllotoxin $0.5 \%$ solution: OR $0.07 ; 95 \%$ CrI 0.001 to 0.36 ) at achieving complete clearance at the end of treatment: results from the primary analysis were supported by sensitivity analysis (table 2). Studies evaluating polyphenon E $10 \%$ ointment were deemed to be at high risk of bias, which precluded their inclusion in the primary analysis of outcomes. Sensitivity analysis found podophyllotoxin $0.5 \%$ solution to be statistically significantly more effective than polyphenon E $10 \%$ ointment (OR $0.03 ; 95 \% \mathrm{CrI}<0.001$ to 0.18 ) at achieving complete clearance at the end of treatment (table 2). No

Table 2 Effect estimates for complete clearance at the end of treatment (selected comparisons) and recurrence vs podophyllotoxin $0.5 \%$ solution

\begin{tabular}{|c|c|c|c|c|}
\hline \multirow[b]{2}{*}{ Outcome } & \multicolumn{2}{|l|}{ Primary analysis } & \multicolumn{2}{|l|}{ Sensitivity analysis } \\
\hline & OR (95\% credible interval) & SUCRA & $\begin{array}{l}\text { OR ( } 95 \% \text { credible } \\
\text { interval) }\end{array}$ & SUCRA \\
\hline Podophyllotoxin $0.5 \%$ solution & N/A & $79.7 \%$ & N/A & $65.0 \%$ \\
\hline Imiquimod 5\% cream & 0.07 (0.001 to 0.36$)$ & $24.1 \%$ & $0.20(0.02$ to 0.75$)$ & $26.3 \%$ \\
\hline $\begin{array}{l}\text { Cryotherapy plus podophyllotoxin } 0.15 \% \\
\text { cream }\end{array}$ & $7.16(0.002$ to 23.97$)$ & $60.1 \%$ & $5.80(0.02$ to 33.74$)$ & $61.2 \%$ \\
\hline Surgical excision & $2.75(0.01$ to 16.31$)$ & $64.2 \%$ & $7.89(0.03$ to 47.47$)$ & $64.5 \%$ \\
\hline $\mathrm{CO}_{2}$ laser therapy & 95.41 (0.04 to 376.50$)$ & $92.3 \%$ & 91.97 (0.30 to 560.3$)$ & $92.0 \%$ \\
\hline Cidofovir & - & & $13.72(0.04$ to 81.66$)$ & $65.3 \%$ \\
\hline \multicolumn{5}{|c|}{ Recurrence (OR >1 favours podophyllotoxin $0.5 \%$ solution) } \\
\hline Imiquimod 5\% cream & $1.76(0.05$ to 9.00$)$ & $54.5 \%$ & N/A & \\
\hline Cryotherapy & $4.42(0.06$ to 22.20$)$ & $33.5 \%$ & N/A & \\
\hline Surgical excision & $0.75(0.007$ to 3.67$)$ & $90.3 \%$ & $\mathrm{~N} / \mathrm{A}$ & \\
\hline
\end{tabular}

$\mathrm{CO}_{2}$, carbon dioxide; N/A, not applicable; OR, odds ratio; SUCRA, surface under the cumulative ranking; TCAA, trichloroacetic acid. 
Rankogram for complete clearance at end of treatment

(primary analysis)

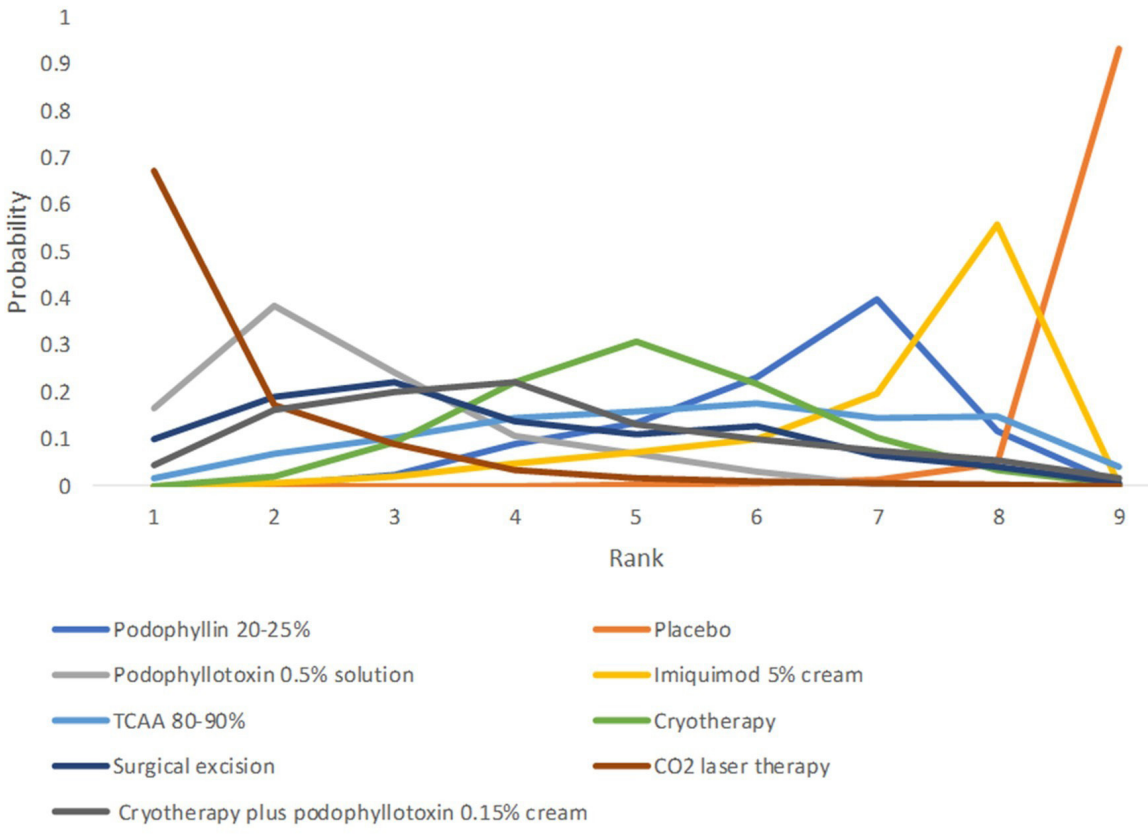

Figure 3 Rankogram for complete clearance at the end of treatment (primary analysis). $\mathrm{CO}_{2}$, carbon dioxide; TCAA, trichloroacetic acid.

other statistically significant differences were identified for other active interventions versus podophyllotoxin $0.5 \%$ solution in either primary or sensitivity analyses. In both the primary and sensitivity analyses, carbon dioxide $\left(\mathrm{CO}_{2}\right)$ laser therapy was found to be the treatment likely to be the ranked first for achieving complete clearance at the end of treatment, with a SUCRA of $92.3 \%$ and 91.7\% in the primary and sensitivity analysis, respectively (table 2). Similarly, in the rankogram (graphical depiction of likelihood of achieving each rank) for the primary analysis of complete clearance at the end of treatment, of the nine interventions forming the network, $\mathrm{CO}_{2}$ laser therapy has the highest probability of being ranked the most effective intervention (figure 3 ). Of the topical treatments assessed in the primary analysis, podophyllotoxin $0.5 \%$ solution was found to have the highest SUCRA and ranking, having the highest probability of being the most effective treatment to achieve complete clearance second to $\mathrm{CO}_{2}$ laser therapy (table 2 and figure 3 ).

Considering complete clearance of AGWs at timepoints after completion of treatment, few identified studies reported clinical effectiveness data on this outcome. Some studies reported follow-up data for the subgroup of people achieving complete clearance at the end of treatment rather than the full study population. Complete clearance without recurrence is distinct from recurrence as the former accounts for people who clear within a few days of completion of treatment. Four studies reporting complete clearance evaluated at least 1 month after end of treatment for all those randomised were included in the NMA. ${ }^{22} 26-28$ Two of the four studies were judged to be of unclear risk of bias, ${ }^{22}{ }^{26}$ with the other two studies deemed to be at a high risk of bias ${ }^{27} 28$ and, therefore, a NMA was feasible for only the preplanned sensitivity analysis. Five interventions were indirectly compared in the NMA: placebo or no treatment, imiquimod $5 \%$ cream (three times a week), cryotherapy, electrotherapy, cryotherapy plus podophyllotoxin $0.15 \%$ cream. ${ }^{11}$ All four active interventions were associated with a statistically significant improvement in complete clearance without recurrence compared with placebo. However, there were no statistically significant differences between any of the active interventions for the outcome. ${ }^{11}$

\section{Recurrence}

For analysis of recurrence, placebo was excluded from the primary NMA due to no people achieving complete clearance after treatment in most identified studies. The primary NMA included five studies, ${ }^{29-33}$ four of which assessed recurrence at 6 months of follow-up, ${ }^{29-31} 33$ and the final study at 3 months after completion of treatment. The network evaluated comparative clinical effectiveness of cryotherapy, imiquimod $5 \%$ cream, podophyllotoxin $0.5 \%$ solution, podophyllin $20 \%$ to $25 \%$ and surgical excision. Random effects model was the better fitting model. When compared with podophyllotoxin $0.5 \%$ solution, the NMA identified no statistically significant difference between any of the comparisons. Assessment of SUCRA indicated that, of the five treatments evaluated, surgical excision was likely to be the most effective treatment for reducing risk of recurrence after complete clearance (table 2). 


\section{Secondary outcomes}

No study was identified in the update reported here that provided new data for the secondary outcomes of volume of wart clearance, appearance of new warts during treatment and adverse effects, which were specified in the protocol. Therefore, re-analysis of the NMA presented in the first iteration of the systematic review was not necessary. ${ }^{11}$ Data on time to complete clearance, relief of symptoms during treatment or quality of life were not identified. Please refer to the original publication for reporting of results for secondary outcomes. ${ }^{11}$

\section{DISCUSSION}

\section{Main findings}

Of the interventions included in the NMA, analysis suggests that $\mathrm{CO}_{2}$ laser therapy is the most effective treatment for achieving complete clearance at the end of treatment. However, the wide CrIs generated in the NMA should be considered when interpreting results. As discussed earlier, although it is widely recognised that ablative techniques, such as $\mathrm{CO}_{2}$ laser therapy, are highly effective at removing AGWs, patient preference is likely to be for a topical treatment that they can apply at home. ${ }^{34}$ Of the topical treatments recommended by BASHH, NMA identified podophyllotoxin $0.5 \%$ solution as having the highest ranking of patient-applied treatments for achieving complete clearance. In terms of recurrence, no statistically significant difference in risk of recurrence at a maximum follow-up of 6 months was found between podophyllotoxin $0.5 \%$ solution and any of cryotherapy, imiquimod $5 \%$ cream and surgical excision, with surgical excision identified as the most effective treatment for reducing risk of recurrence after complete clearance.

\section{Limitations}

A strength of the review is that the evidence base forming the basis of this report was identified through robust systematic review methodology. However, due to their open label nature, about half of the studies identified were judged to be at a high risk of bias, with uncertainty around the quality of the remaining studies due to limited reporting of trial methods in the full text publications. Despite identification of 37 studies, most treatments are compared within only one RCT. Additionally, differences in inclusion criteria were noted across studies, particularly in size and volume of AGWs at baseline, which is likely to result in clinical heterogeneity in evaluated populations and could explain the uncertainty noted in the results of clinical effectiveness, as evidenced by the wide CrIs. Moreover, data for some of the prespecified clinical effectiveness outcomes of interest (eg, relief of symptoms during treatment and quality of life) were not available from any of the identified studies. An additional limitation is lack of a translation for one RCT published in Farsi. ${ }^{21}$ However, it is unclear whether the study was truly randomised and whether the results would influence the findings from the NMA.

\section{CONCLUSIONS}

The implementation of a UK HPV vaccination programme for women that targets the subtypes associated with AGWs has led to a reduction in the number of people diagnosed with AGWs. However, ensuring that the programme is successful is not without its challenges. Not all eligible women will receive the vaccine, and, at this time, the programme does not include boys, and, thus, the need for effective treatments against AGWs remains. With the introduction of the vaccination programme, it is unlikely that further RCTs assessing clinical effectiveness of interventions against AGWs will be carried out, which makes this systematic review timely and highly relevant. Although a large number of RCTs evaluating treatment options for AGWs is available, as a collective, the evidence base is weakened by uncertainty around the quality of studies informing decisions on the effectiveness of individual interventions. Topical treatments that can be applied by the patient are the mainstay of treatment, but the evidence supporting use of imiquimod $5 \%$ cream, podophyllotoxin $0.5 \%$ solution and podophyllotoxin $0.15 \%$ cream is derived from small RCTs that are at a high risk of bias. Moreover, few data are available from direct head-to-head comparisons of treatments. New RCTs assessing comparative clinical effectiveness are unlikely, but, given the considerable psychological burden to the patient with AGW and the cost of treatment of AGWs, it would be beneficial to carry out a high quality study to compare directly the preferred treatment options to better inform decision-making.

Acknowledgements Thanks to Natalie Masento and Charlotta Karner for their contribution to the review of titles and abstracts for relevance of studies to the review. Thanks to Gemma Marceniuk for her contribution to the validation of the network meta-analyses. Thanks also to Elizabeth Thurgar and Dr Nathan Mayura for their contribution to the original piece of research on which this review is based.

Contributors SB and VW reviewed titles and abstracts for inclusion into the review. SB and VW evaluated full publications for inclusion in the review. VW carried out the network meta-analyses, which was validated by SE. SB and VW contributed equally to the drafting of this article. COM contributed clinical expertise throughout the project. SE and COM edited and commented on draft reports and read the final article.

Funding The review reported here is an update and extension to independent research funded by the National Institute for Health Research (NIHR) Health Technology Assessment programme (HTA 12/44). The views and opinions expressed in this report are those of the authors and not necessarily those of the NHS, the NIHR or the Department of Health and Social Care.

Competing interests None declared.

Patient consent for publication Not required.

Provenance and peer review Not commissioned; externally peer reviewed.

Data availability statement All data relevant to the study are included in the article or uploaded as supplementary information.

Open access This is an open access article distributed in accordance with the Creative Commons Attribution Non Commercial (CC BY-NC 4.0) license, which permits others to distribute, remix, adapt, build upon this work noncommercially, and license their derivative works on different terms, provided the original work is properly cited, appropriate credit is given, any changes made indicated, and the use is non-commercial. See: http://creativecommons.org/ licenses/by-nc/4.0/. 


\section{REFERENCES}

1 Patel $\mathrm{H}$, Wagner $\mathrm{M}$, Singhal $\mathrm{P}$, et al. Systematic review of the incidence and prevalence of genital warts. BMC Infect Dis 2013;13:39

2 World Health Organisation. International agency for research on cancer. IARC monograph on the evaluation of carcinogenic risks to humans. Human Papillomaviruses, 2007. https://monographs.iarc.fr/ wp-content/uploads/2018/06/mono90.pdf

3 Muñoz N, Bosch FX, de Sanjosé S, et al. Epidemiologic classification of human papillomavirus types associated with cervical cancer. $N$ Engl J Med 2003;348:518-27.

4 Department of Health. Introduction of human papillomavirus vaccine into the National immunisation programme: announcement of vaccine to be used, 2008. Available: http://webarchive. nationalarchives.gov.uk/20130107105354/http://www.dh.gov.uk/ prod_consum_dh/groups/dh_digitalassets/documents/digitalasset/ dh 085581.pdf [Accessed 24 Oct 2018].

5 Joint Committee on vaccination and immunisation. statement on HPV vaccination, 2018. Available: https://assets.publishing.service. gov.uk/government/uploads/system/uploads/attachment_data/file/ 726319/JCVI_Statement_on_HPV_vaccination_2018.pdf [Accessed 29 Oct 2018].

6 Ali H, Donovan B, Wand H, et al. Genital warts in young Australians five years into national human papillomavirus vaccination programme: national surveillance data. BMJ 2013;346.

7 British association of sexual health and HIV. UK national guidelines on the management of anogenital warts, 2015. Available: https:// www.bashhguidelines.org/media/1075/uk-national-guideline-onwarts-2015-final.pdf [Accessed 29 Nov 2017].

8 Matteelli A, Beltrame A, Graifemberghi S, et al. Efficacy and tolerability of topical $1 \%$ cidofovir cream for the treatment of external anogenital warts in HIV-infected persons. Sex Transm Dis 2001;28:343-6.

9 Orlando G, Fasolo MM, Beretta R, et al. Combined surgery and cidofovir is an effective treatment for genital warts in HIV-infected patients. AIDS 2002;16:447-50.

10 Snoeck R, Bossens M, Parent D, et al. Phase II Double-Blind, Placebo-Controlled study of the safety and efficacy of cidofovir topical gel for the treatment of patients with human papillomavirus infection. Clin Infect Dis 2001;33:597-602.

11 Thurgar E, Barton S, Karner C, et al. Clinical effectiveness and costeffectiveness of interventions for the treatment of anogenital warts: systematic review and economic evaluation. Health Technol Assess 2016;20:1-486.

12 Werner RN, Westfechtel L, Dressler C, et al. Self-Administered interventions for anogenital warts in immunocompetent patients: a systematic review and meta-analysis. Sex Transm Infect 2017;93:155-61.

13 Information Specialists' Sub-Group. The InterTASC Information Specialists' Sub-Group Search Filter Resource. Centre for Reviews and Dissemination, 2017. Available: https://sites.google.com/a/york ac.uk/issg-search-filters-resource/home/search-filters-by-design [Accessed 29 Nov 2017].

14 Centre for reviews and dissemination (CRD). CRD's guidance for undertaking reviews in healthcare, 2011. Available: https://www. york.ac.uk/media/crd/Systematic_Reviews.pdf [Accessed 8 Nov 2018]

15 Higgins JPT, Altman DG, Gotzsche PC, et al. The Cochrane collaboration's tool for assessing risk of bias in randomised trials. BMJ 2011;343:d5928.

16 Higgins JP, Green S. Cochrane handbook for systematic reviews of interventions. Wiley Online Library, 2008.

17 Dias S, Welton NJ, Sutton AJ, et al. Nice dsu technical support document 1: introduction to evidence synthesis for decision making, 2012. Available: http://www.nicedsu.org.uk/TSD1\%20Introduction. final.08.05.12.pdf. [Accessed 7 Mar 2017].

18 Dias S, Welton NJ, Sutton AJ, et al. Nice dsu technical support document 2: a generalised linear modelling framework for pairwise and network meta-analysis of randomised controlled trials, 2016. Available: http://www.nicedsu.org.uk/TSD2\%20General\%20meta\% 20analysis\%20corrected\%202Sep2016v2.pdf [Accessed 7 Mar 2017].

19 Woods BS, Hawkins N, Scott DA. Network meta-analysis on the loghazard scale, combining count and hazard ratio statistics accounting for multi-arm trials: a tutorial. BMC Med Res Methodol 2010;10:54.

20 Salanti G, Ades AE, loannidis JPA. Graphical methods and numerical summaries for presenting results from multiple-treatment metaanalysis: an overview and tutorial. J Clin Epidemiol 2011;64:163-71.

21 Kafaie P, Torabipour M-S. Comparing the efficacy of cryotherapy with $25 \%$ solution of podophyllin in the treatment of genital warts in women. J Isfahan Med School 2015;33:1710-7.
22 Benedetti Panici P, Scambia G, Baiocchi G, et al. Randomized clinical trial comparing systemic interferon with diathermocoagulation in primary multiple and widespread anogenital condyloma. Obstet Gynecol 1989;74:393-7.

23 Lacey CJN, Goodall RL, Ragnarson Tennvall G, et al. Randomised controlled trial and economic evaluation of podophyllotoxin solution, podophyllotoxin cream, and podophyllin in the treatment of genital warts. Sex Transm Infect 2003;79:270-5.

24 Gilson RJC, Shupack JL, Friedman-Kien AE, et al. A randomized, controlled, safety study using imiquimod for the topical treatment of anogenital warts in HIV-infected patients. AIDS 1999;13:2397-404.

25 Stockfleth E, Beti H, Orasan R, et al. Topical Polyphenon ${ }^{\circledR} \mathrm{E}$ in the treatment of external genital and perianal warts: a randomized controlled trial. British Journal of Dermatology 2008;158:1329-38.

26 Gilson RJC, Ross J, Maw R, et al. A multicentre, randomised, double-blind, placebo controlled study of cryotherapy versus cryotherapy and podophyllotoxin cream as treatment for external anogenital warts. Sex Transm Infect 2009;85:514-9.

27 Simmons PD, Langlet F, Thin RN. Cryotherapy versus electrocautery in the treatment of genital warts. Sex Transm Infect 1981;57:273-4.

28 Stefanaki C, Katzouranis I, Lagogianni E, et al. Comparison of cryotherapy to imiquimod $5 \%$ in the treatment of anogenital warts. Int J STD AIDS 2008;19:441-4.

29 Akhavan S, Mohammadi SR, Modarres Gillani M, et al. Efficacy of combination therapy of oral zinc sulfate with imiquimod, podophyllin or cryotherapy in the treatment of vulvar warts. J Obstet Gynaecol Res 2014;40:2110-3.

30 Jensen SL. Comparison of podophyllin application with simple surgical excision in clearance and recurrence of perianal condylomata acuminata. Lancet 1985;2:1146-8.

31 Kar PK, Rajagopal R, Murthy PS. Topical podophyllin and podophyllotoxin for treatment of genital warts : a comparative study. Ind. J. Dermatol 2003;48:146-50.

32 Lassus A, Haukka K, Forsstrom S. Podophyllotoxin for treatment of genital warts in males. A comparison with conventional podophyllin therapy. Eur. J. Sex Transm. Dis 1984;2:31-3.

33 Padhiar BB, Karia UK, Aggarwal R, et al. A comparative study of efficacy of imiquimod $5 \%$ versus podophyllin $20 \%$ in treatment of external and genital warts. Indian J Sex Transm Dis 2006;27:67-9.

34 O'Mahony C, Law C, Gollnick HPM, et al. New patient-applied therapy for anogenital warts is rated favourably by patients. Int J STD AIDS 2001;12:565-70.

35 Arican O, Guneri F, Bilgic K, et al. Topical imiquimod 5\% cream in external anogenital warts: a randomized, double-blind, placebocontrolled study. J Dermatol 2004;31:627-31.

36 Beutner KR, Spruance SL, Hougham AJ, et al. Treatment of genital warts with an immune-response modifier (imiquimod). J Am Acad Dermatol 1998;38:230-9.

37 Edwards L, Ferenczy A, Eron L, et al. Self-administered topical 5\% imiquimod cream for external anogenital warts. HPV Study Group. Human PapillomaVirus. Arch Dermatol 1998;134:25-30.

38 Tyring SK, Arany I, Stanley MA, et al. A randomized, controlled, molecular study of condylomata acuminata clearance during treatment with imiquimod. Journal of Infectious Diseases 1998;178:551-5

39 Tatti S, Swinehart JM, Thielert C, et al. Sinecatechins, a defined green tea extract, in the treatment of external anogenital warts: a randomized controlled trial. Obstet Gynecol 2008;111:1371-9.

40 Beutner K, Friedman-Kien A, Artman N, et al. Patient-applied podofilox for treatment of genital warts. The Lancet 1989;333:831-4.

41 Kirby P, Dunne A, King DH, et al. Double-Blind randomized clinical trial of self-administered podofilox solution versus vehicle in the treatment of genital warts. Am J Med 1990;88:465-9.

42 von Krogh G, Szpak E, Andersson M, et al. Self-treatment using $0.25 \%-0.50 \%$ podophyllotoxin-ethanol solutions against penile condylomata acuminata: a placebo-controlled comparative study. Sex Transm Infect 1994;70:105-9.

43 Greenberg MD, Rutledge LH, Reid R, et al. A double-blind, randomized trial of $0.5 \%$ podofilox and placebo for the treatment of genital warts in women. Obstet Gynecol 1991;77:735-9.

44 Strand A, Brinkeborn RM, Siboulet A. Topical treatment of genital warts in men, an open study of podophyllotoxin cream compared with solution. Sex Transm Infect 1995;71:387-90.

45 Claesson U, Lassus A, Happonen $\mathrm{H}$, et al. Topical treatment of venereal warts: a comparative open study of podophyllotoxin cream versus solution. Int J STD AIDS 1996;7:429-34.

46 Komericki P, Akkilic-Materna M, Strimitzer T, et al. Efficacy and safety of imiquimod versus podophyllotoxin in the treatment of anogenital warts. Sex Transm Dis 2011;38:216-8.

47 Tuncel A, Erbagci MZ, Ozgoztasi O. An open-label comparative study to evaluate the efficacy and tolerability of imiquimod $5 \%$ cream 
alone and combined with cryotherapy in the treatment of recalcitrant anogenital warts. The 14th Congress of the European Academy of Dermatology and Venereology; 2005; London, UK. J Eur Acad Dermtaol Venereol 2005:1-412.

48 Abdullah AN, Walzman M, Wade A. Treatment of external genital warts comparing cryotherapy (liquid nitrogen) and trichloracetic acid. Sex Transm Dis 1993;20:344-5.

49 Stone KM, Becker TM, Hadgu A, et al. Treatment of external genital warts: a randomised clinical trial comparing podophyllin, cryotherapy, and electrodesiccation. Sex Transm Infect 1990;66:16-19.

50 Azizjalali M, Ghaffarpour G, Mousavifard BCO. 2) laser therapy versus cryotherapy in treatment of genital warts; a randomized controlled trial (RCT). Iran J Microbiol 2012;4:187-90.

51 Braga EA, Lopes Filho GdeJ, Saad SS. Argon plasma versus electrofulguration in the treatment of anal and perianal condylomata acuminata in patients with acquired immunodeficiency virus. Acta Cir. Bras. 2017;32:482-90.
52 Ferenczy A, Behelak Y, Haber G, et al. Treating vaginal and external anogenital condylomas with electrosurgery vs $\mathrm{CO} 2$ laser ablation. $J$ Gynecol Surg 1995;11:41-50.

53 Viazis N, Vlachogiannakos J, Vasiliadis K, et al. Earlier eradication of intra-anal warts with argon plasma coagulator combined with imiquimod cream compared with argon plasma coagulator alone: a prospective, randomized trial. Dis Colon Rectum 2007;50:2173-9.

54 Edwards A, Atma-Ram A, Thin RN. Podophyllotoxin 0.5\% $\checkmark$ podophyllin $20 \%$ to treat penile warts. Sex Transm Infect 1988;64:263-5.

55 Mazurkiewicz W, Jablońska S. Clinical efficacy of Condyline $(0.5 \%$ podophyllotoxin) solution and cream versus podophyllin in the treatment of external condylomata acuminata. J Dermatolog Treat 1990;1:123-5.

56 Sherrard J, Riddell L. Comparison of the effectiveness of commonly used clinic-based treatments for external genital warts. Int J STD AIDS 2007;18:365-8. 